\section{Sub-Tenon's local anaesthesia for the treatment of retinopathy of prematurity with diode laser}

\begin{abstract}
Purpose To evaluate the safety and efficacy of sub-Tenon's local anaesthesia (LA) for laser treatment of threshold retinopathy of prematurity (ROP).

Methods A cohort of 18 eyes of 10 neonates
\end{abstract} with threshold ROP was treated with diode laser peripheral retinal ablation over 14 treatment sessions using oral sedation and sub-Tenon's anaesthesia. Treatment was performed in a neonatal unit. The heart and respiratory rate, trans-cutaneous oxygen saturation, and physical activity were continuously monitored for signs of neonatal distress. Cardio-respiratory stability during and after the procedure was scored for each eye treated using a Cardio-respiratory index described previously.

Results In all cases, the planned dose of laser was delivered. One out of 18 eyes developed stage $4 \mathrm{~b}$ tractional retinal detachment over a follow-up of at least 6 months for all babies. Systemic complications during treatment included transient bradycardia and apnoea requiring stimulation in two babies and excessive head mobility in three babies. Treatment duration varied between 20 and $80 \mathrm{~min}$. Ocular complications were conjunctival haemorrhage and chemosis. The mean cardio-respiratory index was 1.13 , which compared favourably with the standard published.

Conclusions Sub-Tenon's LA with oral sedation appears to be a safe and effective alternative to general anaesthesia for laser treatment of ROP, with the potential for lower morbidity.

Eye (2008) 22, 375-379; doi:10.1038/sj.eye.6702642; published online 30 March 2007
MV Parulekar, SDM Chen and CK Patel

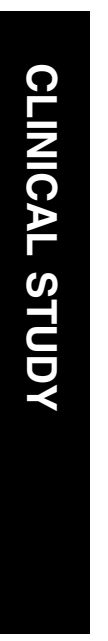

Keywords: retinopathy of prematurity;

sub-Tenons anaesthesia; laser treatment

\section{Introduction}

Retinopathy of prematurity (ROP) is a vasoproliferative disorder affecting the premature retina. Screening for ROP is routinely performed using topical anaesthesia in the neonatal unit. A small proportion of cases screened will progress to threshold ROP, ${ }^{1}$ requiring laser treatment.

There is a wide variation in the anaesthetic techniques used for the laser treatment for threshold retinopathy of prematurity. According to a recent UK survey, general anaesthesia is the most popular, intravenous sedation with intubation the second, and topical anaesthesia, with or without sedation, the least used. $^{2}$

General anaesthesia in premature neonates can be associated with a high risk of morbidity, especially when intraventricular haemorrhage, patent ductus arteriosus, and necrotising enterocolitis are also present.

Topical anaesthesia alone, although adequate for screening examination, is insufficient for treatment and may be associated with an increased incidence of potentially lifethreatening cardio-respiratory events.

We describe our audit of oral sedation with adjunctive Sub-Tenon's anaesthesia as an alternative to general anaesthesia when performing laser treatment for threshold ROP.

Materials and methods

A cohort of 18 eyes of 10 babies with threshold ROP underwent peripheral retinal diode laser
Oxford Eye Hospital,

Oxford, UK

Correspondence: CK Patel, Oxford Eye Hospital,

Woodstock Road,

Oxford

OX2 6HE, UK

Tel: + 441865311 188;

Fax: +44 1865224515 .

E-mail:

ckpatel@btinternet.com

Received: 7 March 2006 Accepted in revised form:

1 October 2006

Published online:

30 March 2007

Proprietary interests: None.

Research funding: None.

Previously presented at British and Eire Association of Vitreo-retinal surgeons (BEAVRS) Annual Meeting, Cheltenham, November 2005.

Submitted to: Oxford Ophthalmological Congress, July 2006 
Table 1 Cardio-respiratory index (from Haigh et al)

\begin{tabular}{ll}
\hline Score & Cardio-respiratory status \\
\hline 0 & Improved from baseline \\
1 & No change \\
2 & Mild instability responding to mild stimulation \\
3 & Marked instability responding to vigorous stimulation \\
4 & Life-threatening event requiring emergency \\
& resuscitation - bagging, suction, intubation \\
\hline
\end{tabular}

photocoagulation using sub-Tenon's local anaesthesia and oral sedation over a total of 14 treatment sessions, and was followed postoperatively for a minimum of 6 months by a consultant surgeon with a subspecialty interest in ROP (ckp).

All treatment sessions were performed in the presence of medical staff at the neonatal unit of the John Radcliffe Hospital, Oxford, England, between December 2002 and October 2005. Operative data were recorded prospectively. The heart and respiratory rates, including oxygen saturation of all neonates are routinely recorded hourly on apnoea and bradycardia charts in our unit. This information was used to derive a cardio-respiratory index (CRI) as described by Haigh et $\mathrm{al}^{4}$ (Table 1). A lower score indicates greater cardiac and respiratory stability during the peri-operative period. The CRI was determined separately for each treated eye, during and for 4 days following surgery.

Written informed parental consent was obtained in all cases. The pupils were dilated with phenylephrine $2.5 \%$ and cyclopentolate $0.5 \%$ eye drops, administered 60 and $30 \mathrm{~min}$ before treatment. Sedation was achieved with chloral hydrate $(50 \mathrm{mg} / \mathrm{kg})$, given orally $1 \mathrm{~h}$ before the procedure. The baby was immobilised by wrapping the arms and body in a sheet, and the head was stabilised by an assistant. The fellow eye was taped shut. After instillation of proxymetacaine $0.5 \%$ drops and insertion of a neonatal lid speculum, the conjunctiva and Tenons capsule in the inferonasal quadrant was incised $2-4 \mathrm{~mm}$ posterior to the limbus to enter the sub-Tenon space. A metal, 26G curved lacrimal cannula (Steriseal, Redditch, England), was passed into this space for approximately $5 \mathrm{~mm}$ before injecting no more than $0.5 \mathrm{ml}$ of lignocaine $1 \%(5 \mathrm{mg} / \mathrm{kg})$. Peripheral retinal laser photocoagulation was performed using the binocular indirect diode laser (Iris Medical Oculight SLx, Iris Medical Instruments, Mountainview, CA, USA) and a 28 dioptre lens to produce a moderate white retinal reaction. The peripheral avascular retina was visualised with scleral indentation using a Flynn indenter.

Respiratory rate, heart rate, transcutaneous oxygen saturation (pulse oxymetry), and physical activity were monitored by a neonatal nurse. All infants had
Table 2 Gestational age and weight at birth and at treatment

\begin{tabular}{lrc}
\hline & At birth & At treatment \\
\hline Gestational age (weeks) & $25^{+2}\left(23^{+4}-27\right)$ & $37^{+2}\left(34^{+5}-43\right)$ \\
Weight $(\mathrm{g})$ & $703(570-830)$ & $2094(915-3270)$ \\
\hline
\end{tabular}

intravenous access but no intravenous medication was used.

\section{Results \\ Cohort}

Eighteen eyes of 10 subjects were treated on 14 different occasions. Two babies had bilateral laser treatment twice and two had a second unilateral treatment. Two babies had unilateral treatment giving a total of 23 eye treatment episodes. The mean weight and gestational age at birth and at treatment are shown in Table 2.

\section{Efficacy}

The number of burns per eye varied between 556 and 2620 (mean 1088) at a power of $270-490 \mathrm{~mW}$ and duration of $200 \mathrm{~ms}$. The treatment times ranged from 20 to $80 \mathrm{~min}$ with a mean of $56 \mathrm{~min}$ per treatment episode. At the time of treatment, two babies were receiving nasal CPAP and three were on low flow oxygen, the remaining five self-ventilating in air (Table 3 ).

In all cases, the planned dose of laser was delivered. All cases were followed up for a minimum of 6 months. During this time, one eye of subject E (Table 3) treated with confluent laser on two occasions (1364 burns at $34+6$ weeks, and 2620 burns at $35+6$ weeks) progressed to Stage $4 b$ ROP requiring vitreolensectomy. ${ }^{5}$ The ROP successfully regressed in all other babies.

\section{Complications}

During treatment, two babies developed transient bradycardia and one developed tachycardia that resolved spontaneously within $60 \mathrm{~s}$ of temporarily stopping treatment. No cardiac instability was seen during the postoperative period.

Two babies developed apnoea during treatment, one while giving the Sub-Tenon's injection. One improved spontaneously and the other responded to bagging with mask and oxygen. Both babies were prone to apnoea and spontaneous desaturation preceding and following treatment. There was no change in frequency or duration of the apnoeic spells after the treatment in both cases. The oxygen flow was increased prophylactically for one baby during treatment, as she was prone to frequent spontaneous desaturations. One baby developed an 


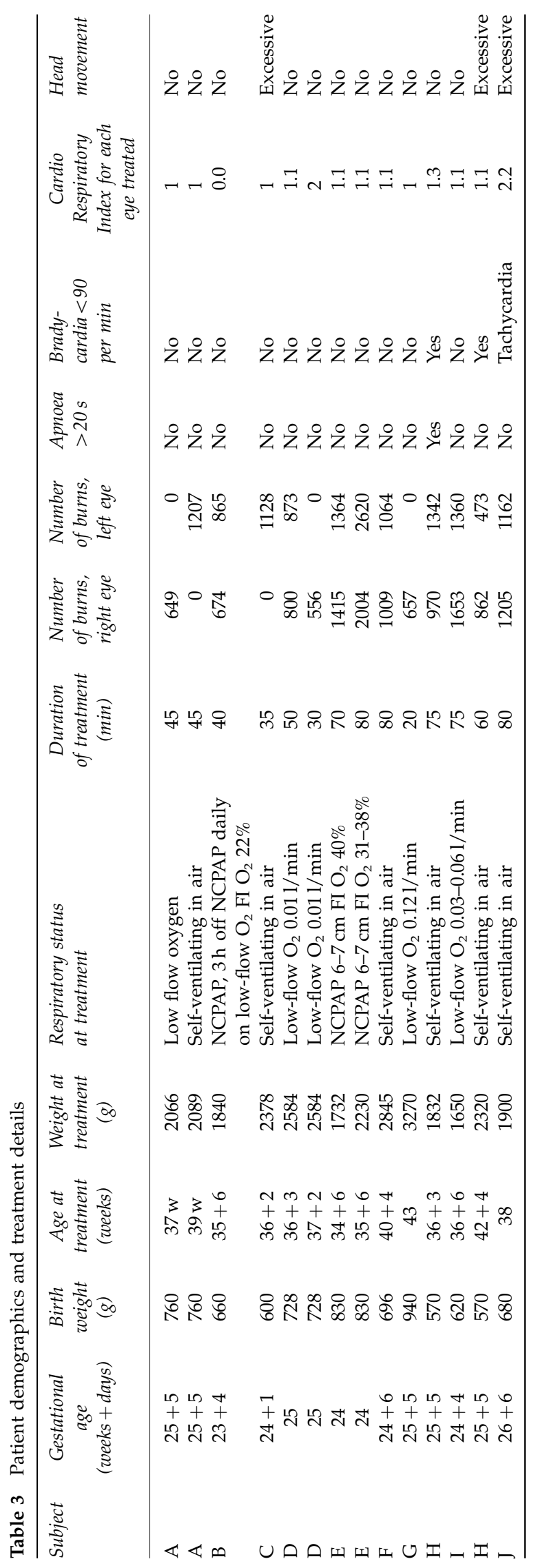

increased oxygen requirement 3 days after treatment for unrelated reasons.

Excessive movement was encountered when treating three babies, two of which settled with a further dose of oral chloral hydrate. In the case where the head movement failed to settle, it was more challenging for the assistant to hold the head still but the treatment was still completed successfully. During all other treatment sessions, signs of neonatal distress as indicated by heart rate, respiratory rate, trans-cutaneous oxygen saturation, and physical activity did not appear to be excessive compared to routine ROP screening examination (Table 3).

There was some degree of subconjunctival haemorrhage and conjunctival chemosis in most cases but did not impair the view or access during treatment. No other ocular or systemic complications were seen related to the laser application or anaesthetic technique.

The mean CRI for each eye treatment episode is shown in Table 3, and the distribution of CRI scores in our series in Figure 1. The mean CRI over 4 days was 1.13. This compares favourably to the published values of 1.08 for intravenous sedation with intubation, and 1.25 for general anaesthesia, and was better than topical anaesthesia with a CRI of 1.98 (Table 4).

\section{Discussion}

Threshold ROP typically develops between 34 and 42 weeks post gestational age. ${ }^{1}$ The incidence and severity of disease increases with decreasing gestational age and birth weight. Premature babies have a very poor respiratory drive and are prone to prolonged and persistent apnoea. ${ }^{6}$ Respiratory support using mechanical ventilation (SIMV) is often required for the initial period, followed by weaning off to nasal CPAP and nasal supplemental oxygen. The weaning off occurs by $34-36$ weeks in most cases. Most of the babies are thus self-ventilating (with supplemental oxygen or nasal

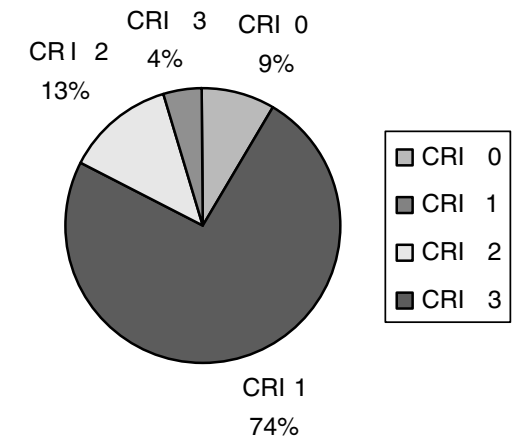

Figure 1 Distribution of (CRI) over 23 eye treatment episodes in our series. 
Table 4 CRI for different anaesthetic techniques

\begin{tabular}{lcc}
\hline Anaesthetic technique & Mean CRI & Adverse events \\
\hline GA & 1.25 & $2 / 12(16 \%)$ \\
i.v. sedation + intubation & 1.08 & $0 / 12(0 \%)$ \\
Topical & 1.98 & $4 / 12(33 \%)$ \\
Sub-Tenon's (Oxford) & 1.13 & $2 / 23(8.7 \%)$ \\
\hline
\end{tabular}

CPAP) around the time acute ROP develops. Their cardio-respiratory system however continues to be fragile, and spontaneous bradycardia and apnoeic spells are common. Any intervention or intercurrent illness can aggravate cardio-respiratory instability. For these reasons, we believe that treatment under local anaesthesia can be performed without a paediatric anaesthetist with the caveat that medical staff should be present on the neonatal unit to intubate babies if the neonatal nurse supporting the ophthalmologists is unable to deal with cardio-respiratory complications.

Laser treatment is the standard modality for the management of acute threshold ROP. This involves manipulation of the globe in the form of lid retraction and scleral indentation. Although laser treatment may not necessarily be painful, the bright light and manipulation of the globe could be stressful and painful for the baby, even if a topical anaesthetic has been administered. Oxygen saturation significantly decreases and pulse rate significantly increases during physical manipulation of the eye, and variations in these parameters may be associated with significant neonatal distress. ${ }^{7-9}$

There is a wide variation between different neonatal units in the methods and availability of anaesthetic techniques used for ROP treatment. ${ }^{4}$ A recent survey of UK ophthalmologists participating in the treatment of acute ROP showed that the majority preferred general anaesthesia $(50 \%)$ or intravenous sedation with intubation $(37 \%){ }^{2}$ General anaesthesia or intravenous sedation is preferred to the use of topical anaesthesia alone because topical anaesthesia may not adequately anaesthetise the eye, resulting in considerable systemic distress. ${ }^{4}$ Topical anaesthesia does not produce ocular akinesia, which may hinder the accurate placement of laser burns and increase the risk of sight-threatening foveal burns.

General anaesthesia or intravenous sedation however requires the presence of neonatal anaesthetic staff. ${ }^{10} \mathrm{In}$ units where trained neonatal anaesthetic staff may not readily be available, significant delays in treatment may occur while arrangements are being made. Apart from the logistic difficulties, these premature babies have a very poor respiratory drive and can be very difficult to wean off the ventilator after intubation for general anaesthesia. This increases morbidity and may unnecessarily prolong their stay on the neonatal unit after laser treatment.

Another technique described involves intravenous infusion of morphine for sedating babies for laser treatment. Morphine is a respiratory depressant and may not be suitable for babies with a poor respiratory drive. It may also cause pupillary miosis, impairing the view of the retina. ${ }^{11}$

We developed the technique of sub-Tenon's LA for laser treatment of ROP in an attempt to reduce the potential distress and systemic risks associated with treatment, while avoiding the need for general anaesthesia. Sub-Tenon's LA has been shown to provide effective analgesia during pan retinal laser photocoagulation in adults. ${ }^{12}$ It dampens the oculocardiac reflex, minimising any tendency to apnoea or bradycardia during and for a few hours after treatment. ${ }^{8}$ An additional benefit of the Sub-Tenon's anaesthetic is augmentation of pupillary dilatation. The pupils are often resistant to pharmacological dilatation in acute ROP. This is believed to be due to engorgement of iris vasculature, and can sometimes pose additional difficulty during treatment.

Lignocaine has been used safely in infants and children for topical, regional, and spinal anaesthesia, without any systemic toxicity. ${ }^{13}$ However, metabolism and elimination of local anaesthetic agents may occasionally be delayed in neonates, and there have been rare reports of methaemoglobinemia and recurrent seizures in young infants following topical and intravenously administered lignocaine. ${ }^{14,15}$ The maximum recommended safe dosage of lignocaine without adrenaline in children is $7 \mathrm{mg} / \mathrm{kg} .{ }^{13}$ We used a maximum dose of $5 \mathrm{mg} / \mathrm{kg}$ and a volume not exceeding $0.5 \mathrm{ml}$.

Other potential complications associated with the sub-Tenon's injection technique in infants include trauma to the optic nerve, extra-ocular muscles, or vortex veins. This is believed to be due to a smaller orbital volume in neonates. We used a modified technique where the injection cannula was passed only a short distance past the equator of the globe (up to $5 \mathrm{~mm}$ ) in order to avoid these risks. A customised cannula for use in neonates would be useful and is being developed.

The sub-Tenon's anaesthesia and laser treatment appeared to be extremely well tolerated in our series. Excessive movement was encountered on three occasions $(21 \%)$ and was overcome with additional oral chloral hydrate or immobilisation of the head by the assistant. Bradycardia and apnoea were seen in a small number of cases $(21 \%)$, one of which required intervention with oxygen mask and bagging. The other two recovered without intervention within $60 \mathrm{~s}$. One case developed transient tachycardia. 
Adequate confluent laser treatment could be performed without interruption in all our cases, with complete resolution of the neovascular process in all but one case $(5.6 \%)$. One eye of subject $E$ with aggressive disease progressed to stage $4 \mathrm{~b}$ despite confluent laser ablation of the peripheral avascular retina and underwent vitreolensectomy. The other eye stabilized after two treatment sessions. This is in keeping with previous series, which have reported progression to stage 4and 5 despite adequate confluent laser treatment in $44.8 \%$ of cases with zone I ROP, and $3.9 \%$ of anterior zone II ROP. ${ }^{16}$

The cardio-respiratory index during the peri-operative period compared favourably with other established anaesthetic techniques in the previously reported series by Haigh et al (Table 4 ).

In conclusion, the results of this series demonstrate that sub-Tenon's local anaesthesia with oral sedation is a safe and effective technique for laser treatment of threshold ROP. We believe that it offers a safe and effective alternative to general or topical anaesthesia with or without intravenous sedation, with considerably lower morbidity. There is scope for further improvement in maintaining head stability during treatment either by improved sedation or head fixation.

\section{References}

1 The royal college of ophthalmologists, the british association of perinatal medicine. Report of a joint working party: guidelines for screening and treatment. Early Human Dev 1996; 46: 239-258.

2 Chen SD, Sundaram V, Wilkinson A, Patel CK. Variation in anaesthesia for the lasertreatment of retinopathy of prematurity - a survey of ophthalmologists in the UK. Eye 2006 (advance online publication 28 July 2006; doi: 10.1038/ sj.eye.6702499).
3 Welborn LG, Greenspun JC. Anesthesia and apnea. Perioperative considerations in the former preterm infant. Pediatr Clin Am 1994; 41(1): 181-198 (Review).

4 Haigh PM, Chiswick ML, O'Donoghue EP. Retinopathy of prematurity: systemic complications associated with different anaesthetic techniques at treatment. $\mathrm{Br} \mathrm{J}$ Ophthalmol 1997; 81: 283-287.

5 Patel CK. Optical coherence tomography in the management of acute retinopathy of prematurity. Am J Ophthalmol 2006; 141: 582-584.

6 Sims C, Johnson CM. Postoperative apnoea in infants. Anaesth Intensive Care 1994; 22: 40-45.

7 Rush R, Rush S, Nicolau J, Chapman K, Naqvi M. Systemic manifestations in response to mydriasis and physical examination during screening for retinopathy of prematurity. Retina 2004; 24: 242-245.

8 Clarke WN, Hodges E, Noel LP, Roberts D, Coneys M. The oculocardiac reflex during ophthalmoscopy in premature infants. Am J Ophthalmol 1985; 99: 649-651.

9 Laws DE, Morton C, Weindling M, Clark D. Systemic effects of screening for retinopathy of prematurity. $\mathrm{Br} J$ Ophthalmol 1996; 80: 425-428.

10 Keenan RL, Shapiro JH, Kane FR, Simpson PM. Bradycardia during anesthesia in infants. An epidemiologic study. Anesthesiology 1994; 80(5): 976-982.

11 Tobias JD. Sedation and analgesia in the pediatric intensive care unit. Pediatr Ann 2005; 34(8): 636-645.

12 Stevens JD, Foss AJ, Hamilton AM. No-needle one-quadrant sub-tenon anaesthesia for panretinal photocoagulation. Eye 1993; 7: 768-771.

13 Fraunfelder FT. Drug Induced Ocular Side Effects and Drug Interactions, 3rd ed. Lea \& Febiger: Philadelphia, 1989.

14 Gonzalez del Rey J, Wason S, Druckenbrod RW. Lidocaine overdose: another preventable case? Pediatr Emerg Care 1994; 10: 344-346.

15 Resar LM, Helfaer MA. Recurrent seizures in a neonate after lidocaine administration. J Perinatol 1998; 18: 193-195.

16 Fallaha N, Lynn MJ, Aaberg Jr TM, Lambert SR. Clinical outcome of confluent laser photoablation for retinopathy of prematurity. Journal of American Association of Pediatric Ophthalmology and Strabismus 2002; 6(2): 81-85. 